\title{
MODELLING OF MULTI-BEARING ROTOR SYSTEMS FOR VIBRATION ANALYSIS AND CONTROL LAW SYNTHESIS
}

\author{
J. M. KRODKIEWSKI \\ L. SUN \\ Department of Mechanical and Manufacturing Engineering \\ The University of Melbourne, Melbourne, Victoria, AUSTRALIA
}

\begin{abstract}
A complete mathematical model for a multi-bearing rotor system incorporating a newly developed active journal bearing has been presented. Both a non-linear model and a general linearization method have been developed. The system configuration parameters (the relative positions of the bearings) were expressed explicitly in the equations of motion. Therefore it makes it convenient to carry out dynamic analysis of the rotor system in either the rotating speed domain or the system configuration parameter domain. The Guyan matrix condensation technique has been adopted to reduce the number of equations in the equations of motion. The developed mathematical model has been used in predicting the critical speeds, thresholds of instability and unbalance response of a multi-bearing rotor system.
\end{abstract}

\section{Introduction}

Increasing demands for high performance rotating machinery have made the rotor dynamic problems more and more complex, and more and more attention has been drawn on rotordynamics. Modelling and computer simulation technology have been widely used in designing and analysing rotor-bearing systems.

A multi-bearing rotor system is statically indeterminate. Its dynamic behaviour depends on the relative positions of the bearings as well as the properties of its subsystems. The relative positions of bearings are usually referred to as system configuration, or bearing alignment (Parszewski and Krodkiewski, [1]). Therefore, the 
dynamic properties of a multi-bearing rotor system are a function of the rotating speed and the system configuration. Consequently, the investigation of the system dynamic behaviour can be undertaken in two domains. One is in the rotating speed domain, where the analysis of the threshold speed of instability and the critical speeds of the system is the major concern. The other is in the configuration domain, where the analysis of the system properties, at the operating speed, as a function of the bearing alignment is the main interest.

The dynamics of a rotor system supported by fluid film bearings is inherently a nonlinear problem. Both linearized methods and non-linear approaches have been used in the modelling and solving rotor dynamic problems. Linearized models are commonly used in predictions of critical speeds, vibration response and instability threshold in a large range of operating points. Non-linear models are used not only to verify results obtained from linearized model, but also to study some important rotor-bearing dynamic phenomena. These phenomena such as sub-harmonic resonance and limit cycles cannot be observed without accounting for highly non-linear forces produced by fluid-film bearings under large amplitude vibrations. Lund [2] used a linearized model to calculate the threshold speed of instability and damped critical speeds of a flexible rotor supported by journal bearings. Adams [3] used a non-linear model to simulate the response of a multi-bearing rotor system.

In order to develop high performance rotating machinery, active vibration control has been paid growing attentions to improve the system dynamic properties by employing active devices. These active devices include: magnetic bearings (Schweitzer, [4]), piezoelectric bearing pushers (Palazzolo et al., [5], Ulbrich and Althaus, [6]), hydraulic actuator journal bearings (Ulbrich and Althaus, [6], [7]), variable impedance bearings (Feng and Xin, [8], Goodwin et al., [9,10]), damper using electro-rheological fluids (Nikolajsen and Hoque, [11]), deformable bushes (Kicinski and Materny, [12]), and active journal bearing with a flexible sleeve (Krodkiewski and Sun, [13]).

This paper presents a modelling technique of multi-bearing rotor system incorporating the newly developed active journal bearing presented by Krodkiewski and Sun [13]). Both a general non-linear model and a linearization method are presented with numerical solutions and simulations.

\section{Description of the active journal bearing.}

The flexible sleeve can be considered as a new feature of the proposed active journal bearing as shown in Fig. 1. The sleeve is activated by the chamber pressure $p_{c}$ which is controlled by valves in the hydraulic system. The oil film of the bearing is separated from the pressure chamber by the flexible seal. Therefore, the chamber pressure will not influence the boundary conditions of the oil film.

The deformation of the flexible sleeve can be changed by adjusting the chamber pressure. Therefore the geometry and thickness of the oil film, and hence the dynamic properties of the rotor system, can be controlled without stopping the operation of the machine. The chamber pressure can also be changed dynamically by a servo valve. So the active journal bearing can deliver dynamic control forces to the rotor via the oil film to control the forced vibration by either an open-loop means or feedback approaches. 


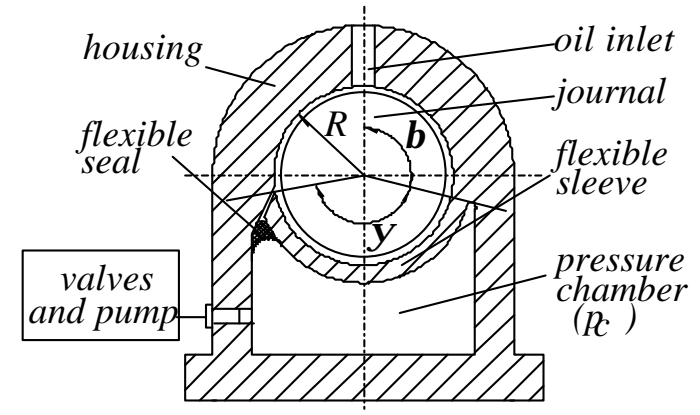

Fig. 1 Schematic of the active journal bearing

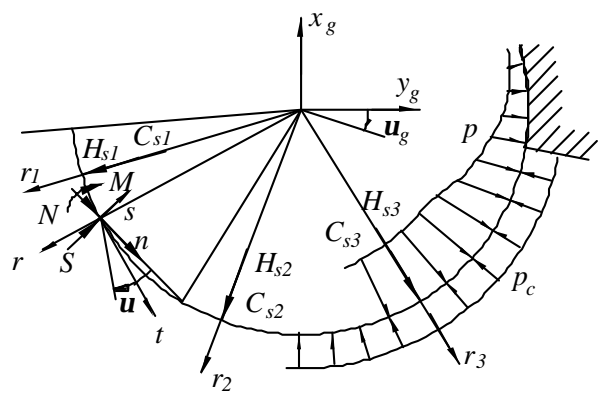

Fig. 2 Model of the flexible sleeve

\section{Modelling of the active journal bearing}

\subsection{Modelling of the Flexible Sleeve}

To obtain the stiffness and mass matrices of the flexible sleeve, the finite element method (FEM) was employed. The flexible sleeve was considered as a curved cantilever beam. Each node has three degrees of freedom (DOF) $s, n, v$, which correspond to nodal forces $\mathbf{S}, \mathbf{N}$ and $\mathbf{M}$ as shown in Fig. 2. By assembling the individual elements along the global co-ordinates $x_{g}, y_{g}$ and $v_{g}$, the initial model of the flexible sleeve was obtained.

In order to reduce the number of DOF, the Guyan reduction technique (Guyan, [14]) was used to condense the mass and stiffness matrices. The condensation was performed in three steps. First, the original matrices were condensed along coordinates $x_{g}$ and $y_{g}$ (the angular co-ordinates were eliminated). Then, the matrices were transferred to system of co-ordinates $r, t$. Finally, the matrices were condensed to a final dimension containing only a few normal co-ordinates.

After the condensed mass and stiffness matrices are obtained, the equations of motion of the flexible sleeve can be adopted as follows:

$$
\mathbf{M s}_{\mathrm{s}} \cdot \ddot{\mathbf{r}}_{\mathrm{s}}+\mathrm{K}_{\mathrm{s}} \cdot \mathbf{r}_{\mathrm{s}}=\mathbf{H}_{\mathrm{s}}+\mathbf{C}_{\mathrm{s}}
$$

where $\mathbf{r}_{\mathbf{s}}$ is vector of the retained co-ordinates. $\mathbf{M}_{\mathbf{s}}$ and $\mathbf{K}_{\mathbf{s}}$ stand for the condensed mass and stiffness matrices. $\mathbf{H}_{\mathbf{s}}$ and $\mathbf{C}_{\mathbf{s}}$ represent the hydrodynamic forces due to the instantaneous oil film pressure $p$ and the chamber pressure $p_{c}$ respectively.

\subsection{Modelling of the Hydrodynamic Forces}

The configuration of the active bearing is shown in Fig. 3. The pressure distribution of the oil film is a function of the instantaneous displacements and velocities of the flexible sleeve and the journal, as well as the rotating speed of the rotor, i.e., $p=p\left(\Omega, \mathbf{r}_{\mathrm{s}}, \mathbf{q}, \dot{\mathbf{r}}_{\mathrm{s}}, \dot{\mathbf{q}}\right)$. The Reynolds equation was used to model the pressure distribution:

$$
\frac{1}{R^{2}} \frac{\partial}{\partial \varphi}\left(\frac{h^{3}}{\eta} \frac{\partial p}{\partial \varphi}\right)+\frac{\partial}{\partial z}\left(\frac{h^{3}}{\eta} \frac{\partial p}{\partial z}\right)=6 \Omega \frac{\partial h}{\partial \varphi}+12 \frac{\partial h}{\partial t}
$$

The thickness of the oil film can be written as:

$$
h=h^{\prime}+\Delta h
$$


where $\Delta h$ is the deformation of the sleeve which can be obtained by solving Eq. 1 . (The full motion of the flexible sleeve is calculated by a co-ordinate transformation provided by the Guyan method, after the solution of Eq. 1 is obtained). $h^{\prime}$ is the thickness disregarding the deformation of the flexible sleeve which can be approximated by:

$$
h^{\prime}=c+q \cdot \cos (\varphi-\alpha)
$$

where $c$ is the bearing radial clearance, $q$ and $\alpha$ are the polar co-ordinates of the centre of the journal.

Then,

$$
\frac{\partial h}{\partial t}=\frac{\partial q}{\partial t} \cos (\varphi-\alpha)+q \cdot \frac{\partial \alpha}{\partial t} \sin (\varphi-\alpha)+\frac{\partial \Delta h}{\partial t}
$$

The integration of Eq. 2 produces the oil film pressure distribution $p$. The principle of virtual work applied to the instantaneous oil pressure results in the hydrodynamic forces $\mathbf{H}_{\mathbf{s}}$ acting on the flexible sleeve. The same principle applied to the chamber pressure $p_{c}$ yields the force vector $\mathbf{C}_{\mathbf{s}}$.

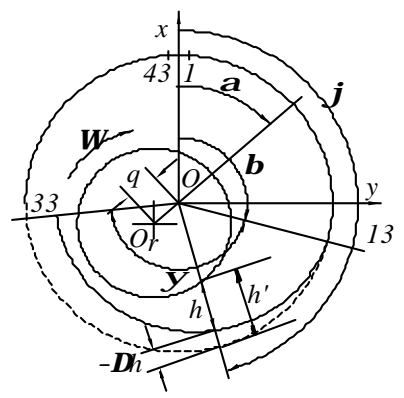

Fig. 3 Configuration of the active bearing

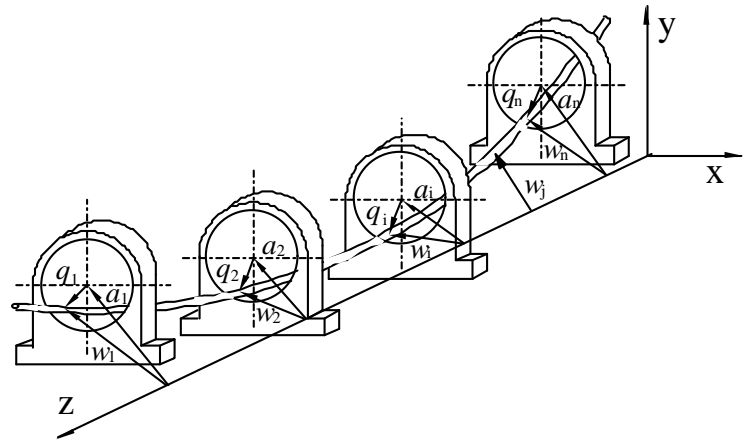

Fig. 4 A multi-bearing-rotor system with rigid supports

\section{Modelling of multi-bearing systems with the active bearing}

Fig. 4 illustrates a multi-bearing rotor system with a rigid foundation. The system is statically indeterminate. Its configuration is defined by vector a. The rotor was treated as a free-free body and was modelled by the FEM using Timoshenko beam elements. After matrix condensation using the Guyan method, the equations of motion for the transverse vibration of the rotor can be expressed in the form,

$$
M \mathbf{M} \cdot \ddot{\mathbf{W}}+K_{\mathbf{r}} \cdot \mathbf{W}=\mathbf{H r}+Q_{\mathbf{r}}+\mathbf{F r}
$$

where $\mathbf{w}$ is the absolute position vector of the rotor nodes. $\mathbf{M}_{\mathbf{r}}$ and $\mathbf{K}_{\mathbf{r}}$ are the mass and stiffness matrices of the rotor. $\mathbf{Q}_{\mathbf{r}}$ and $\mathbf{F}_{\mathbf{r}}$ are static load vector and external excitation forces acting on the rotor respectively. $\mathbf{H}_{\mathbf{r}}$ is the vector of hydrodynamic forces from the oil film.

The absolute displacements at the nodes of rotor where bearings are present can be expressed as,

$$
w_{i}=q_{i}+a_{i} \quad i=1,2, \ldots, m(m-\text { the number of bearings })
$$


whereas for all the other nodes,

$$
w_{j}=q_{j}
$$

then, vector $\mathbf{w}$ can be expressed as,

$$
\mathbf{w}=\mathbf{q}+\mathbf{a}
$$

The elements of vector a are zero for the nodes where there is no bearing. Substituting Eq. 9 into Eq. 6, we have,

$$
\mathbf{M r}_{\mathbf{r}} \cdot \ddot{\mathbf{q}}+\mathbf{K}_{\mathbf{r}} \cdot \mathbf{q}=\mathbf{H r}+\mathbf{Q r}_{\mathbf{r}}-\mathbf{K}_{\mathbf{r}} \cdot \mathbf{a}+\mathbf{F}_{\mathbf{r}}(10)
$$

When the active bearing is present in the system, the hydrodynamic force $\mathbf{H}_{\mathbf{r}}$ and $\mathbf{H}_{\mathbf{s}}$ (in Eq. 1) are a non-linear function of the motion of both the journal and the flexible sleeve of the bearing. Therefore, Eq. 1 and Eq. 10 form a simultaneous set of nonlinear differential equations coupled by the hydrodynamic forces from oil films governed by Eq. 2.

The hydraulic force from the pressure chamber $\mathbf{C}_{\mathrm{s}}$ in Eq. 1 could be divided into a constant part, $\mathbf{C}_{\mathbf{s} 0}$, and a fluctuating part $\Delta \mathbf{C}_{\mathbf{s}}$ over the constant component, i.e.

$$
\mathbf{C}_{\mathrm{s}}=\mathbf{C}_{\mathrm{s} 0}+\Delta \mathbf{C}_{\mathrm{s}}
$$

The equations of motion of the system, Eq. 1 and Eq. 10, can be written in a compact form,

$$
\mathbf{M} \cdot \ddot{\mathbf{r}}+\mathbf{K} \cdot \mathbf{r}=\mathbf{H}+\mathbf{Q}+\mathbf{F}+\mathbf{C}_{\mathbf{0}}+\Delta \mathbf{C}-\mathbf{K} \cdot \mathbf{a}_{\mathbf{r}}
$$

where

$$
\begin{aligned}
& \mathbf{M}=\left[\begin{array}{cc}
\mathbf{M}_{\mathbf{r}} & \mathbf{0} \\
\mathbf{0} & \mathbf{M}_{\mathrm{s}}
\end{array}\right], \quad \mathbf{K}=\left[\begin{array}{cc}
\mathbf{K}_{\mathbf{r}} & \mathbf{0} \\
\mathbf{0} & \mathbf{K}_{\mathrm{s}}
\end{array}\right], \quad \mathbf{r}=\left[\begin{array}{c}
\mathbf{q} \\
\mathbf{r}_{\mathrm{s}}
\end{array}\right], \quad \mathbf{H}=\left[\begin{array}{l}
\mathbf{H}_{\mathbf{r}} \\
\mathbf{H}_{\mathrm{s}}
\end{array}\right] \\
& \mathbf{Q}=\left[\begin{array}{c}
\mathbf{Q}_{\mathbf{r}} \\
\mathbf{0}
\end{array}\right], \quad \mathbf{F}=\left[\begin{array}{c}
\mathbf{F}_{\mathbf{r}} \\
\mathbf{0}
\end{array}\right], \quad \mathbf{C}_{\mathbf{0}}=\left[\begin{array}{c}
\mathbf{0} \\
\mathbf{C}_{\mathrm{s} \mathbf{0}}
\end{array}\right], \quad \Delta \mathbf{C}=\left[\begin{array}{c}
\mathbf{0} \\
\Delta \mathbf{C}_{\mathrm{s}}
\end{array}\right], \quad \mathbf{a}_{\mathbf{r}}=\left[\begin{array}{l}
\mathbf{a} \\
\mathbf{0}
\end{array}\right]
\end{aligned}
$$

$\mathbf{H}$ refers to the hydrodynamic forces. It is a function of the rotating speed and the motion of the rotor and the flexible sleeve, i.e. $\mathbf{H}=\mathbf{H}(\Omega, \mathbf{r}, \dot{\mathbf{r}}) . \mathbf{Q}$ refers to the static forces, $\mathbf{F}$ the external excitation forces, $\mathbf{C}_{\mathbf{0}}$ and $\Delta \mathbf{C}$ the static and dynamic control forces from the pressure chamber, $\mathbf{K} \mathbf{a}_{\mathbf{r}}$ the forces caused by the relative positions of bearings, or the system configuration parameters, due to the statically indeterminate property.

Eq. 12 can be solved by numerical integration methods. At each time interval the Reynolds equation 2 is solved numerically e.g. by the finite element method or the finite difference method. The authors used the Runge-Kutta method for the numerical integration and finite difference method for solution of Eq. 2. As it can be expected, one solution at one operating point generally requires a considerable time using these numerical methods based on the non-linear model. 


\section{Linearization of the equations of motion}

A linearized model is advantageous in predicting dynamic performance of a non-linear system in a comprehensive range of operation, as solutions based on a non-linear model is generally very time consuming.

The non-linearity in Eq. 12 is caused by the non-linear properties of the hydrodynamic forces of the oil film with respect to the motion of the system. The linearization of Eq. 12 can be performed by linearizing these forces in a vicinity of an equilibrium position. Therefore solutions of the equilibrium position is essential for the linearization procedure.

The steady state equilibrium position of the system can be found by solving the following static equations,

$$
\mathbf{K} \cdot \mathbf{r}=\mathbf{H}(\Omega, \mathbf{r}, \mathbf{0})+\mathbf{Q}+\mathbf{C}_{\mathbf{0}}-\mathbf{K} \cdot \mathbf{a}_{\mathbf{r}}(14)
$$

These equations are non-linear and implicit, and can only be solved by numerical methods. The equations are not a polynomial type. So that a successive substitution method is suitable to solve it numerically (Carnahan et al., [15]). The recursive procedure is defined by

$$
\mathbf{K} \cdot \mathbf{r}_{j+1}=\mathbf{H}\left(\Omega, \mathbf{r}_{j}, \mathbf{0}\right)+\mathbf{Q}+\mathbf{C}_{\mathbf{0}}-\mathbf{K} \cdot \mathbf{a}_{\mathbf{r}}
$$

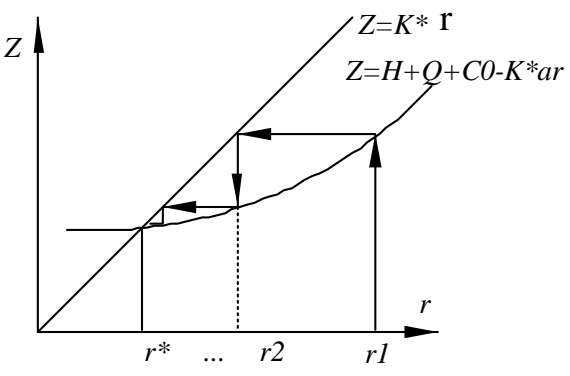

(a)

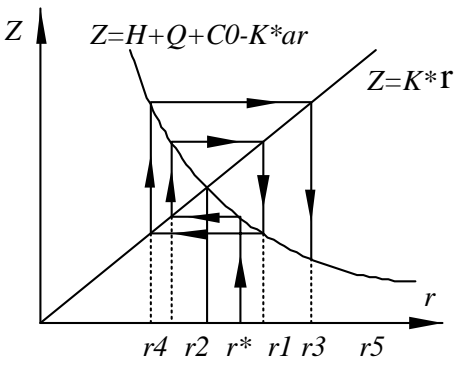

(b)

Fig. 5 Graphical interpretation of the successive substitution method

The successive iterations are interpreted graphically in Fig. 5 ( $\boldsymbol{r}^{*}$ refers to the solution of Eq. 14). Convergence will certainly occur in the case of Fig. 5(a). While in the case of Fig. 5(b), the iterations of Eq. 15 will fail to converge to solution $\boldsymbol{r}^{*}$. Some modifications of Eq. 15 can be made to bring the successive substitutions successful (Traub, [16]). The modification which is made here is as follows.

$$
\mathbf{K} \cdot \mathbf{r}+P_{m} \cdot \mathbf{K} \cdot \mathbf{r}=P_{m} \cdot \mathbf{K} \cdot \mathbf{r}+\mathbf{H}(\Omega, \mathbf{r}, \mathbf{0})+\mathbf{Q}+\mathbf{C}_{\mathbf{0}}-\mathbf{K} \cdot \mathbf{a}_{\mathbf{r}}
$$

$P_{m}$ is a coefficient to be chosen to make the iterations convergent. The authors have found that by choosing a proper value of $P_{m}$, the iterations can always converge to an equilibrium position.

After an equilibrium position is found, the linearization of the equations of motion is achieved by linearizing the hydrodynamic forces in a vicinity of the equilibrium position by perturbation methods. The displacement vector and the hydrodynamic forces can be written in the following forms in a vicinity of an equilibrium position,

$$
\mathbf{r}=\mathbf{r}_{\mathbf{0}}+\Delta \mathbf{r}, \quad \mathbf{H}=\mathbf{H}_{\mathbf{0}}+\Delta \mathbf{H}
$$


where $\mathbf{r}_{\mathbf{0}}$ refers to the equilibrium position ( $\boldsymbol{r}^{*}$ in Fig. 5), and $\mathbf{H}_{\mathbf{0}}$ the hydrodynamic forces when the rotor system is at the equilibrium position.

$\Delta \mathbf{H}$ is assumed to be linearly proportional to the displacements and velocities of the system in a vicinity of the equilibrium position, that is,

$$
\Delta \mathbf{H}=-\mathbf{K}_{\mathbf{H}} \cdot \Delta \mathbf{r}-\mathbf{D}_{\mathbf{H}} \cdot \Delta \dot{\mathbf{r}}
$$

where $\mathbf{K}_{\mathbf{H}}$ and $\mathbf{D}_{\mathbf{H}}$ are the matrices of the coefficients, which can be defined as stiffness and damping matrices of the oil films. Introducing Eq. 17 and Eq. 18 into Eq. 12, and taking the condition of the equilibrium position (Eq. 14) into account, we have the linearized equations of motion of the rotor-bearing system,

$$
\mathbf{M} \cdot \Delta \ddot{\mathbf{r}}+\mathbf{D}_{\mathbf{H}} \cdot \Delta \dot{\mathbf{r}}+\left(\mathbf{K}+\mathbf{K}_{\mathbf{H}}\right) \cdot \Delta \mathbf{r}=\mathbf{F}+\Delta \mathbf{C}
$$

The elements of matrices $\mathbf{K}_{\mathbf{H}}$ and $\mathbf{D}_{\mathbf{H}}$ can be approximated by the following finite difference calculation,

$$
\begin{array}{ll}
\left(K_{H}\right)_{i, j}=-\frac{H_{i}\left(\Omega, \mathbf{r}_{\mathbf{0}}+\Delta \mathbf{r}, \mathbf{0}\right)-H_{i}\left(\Omega, \mathbf{r}_{\mathbf{0}}, \mathbf{0}\right)}{\Delta r_{j}}, & i=1,2, \cdots, n, \\
\left(D_{H}\right)_{i, j}=-\frac{H_{i}\left(\Omega, \mathbf{r}_{\mathbf{0}}, \Delta \dot{\mathbf{r}}\right)-H_{i}\left(\Omega, \mathbf{r}_{\mathbf{0}}, \mathbf{0}\right)}{\Delta \dot{r}_{j}}, & j=1,2, \cdots, n,
\end{array}
$$

where $\mathbf{r}_{\mathbf{0}}+\Delta \mathbf{r}$ is defined in such a way that only the $j$-th element of the vector $\mathbf{r}$ is changed by $\Delta r_{j}$ with respect to the equilibrium position $\mathbf{r}_{\mathbf{0}}$. By the same way, $\Delta \dot{\mathbf{r}}$ implies that the value of the $j$-th element of the vector $\dot{\mathbf{r}}$ is $\Delta \dot{r}_{j}$, and all the others are zero. $n$ is the number of degrees of freedom in the equations of motion, Eq. 12.

Stability analysis can be performed by solving the eigenvalue problem of Eq. 19. The forced responses are solved under certain excitation forces, normally the centrifugal forces due to imbalance of the rotor, and the control forces. The equations can also been used for synthesis of control laws for various control strategies.

The solution of equilibrium position is essential in the linearization procedure. The stiffness and damping coefficients of the oil films are a function of the equilibrium position. Therefore, the dynamic characteristic of the system is a function of the equilibrium position.

\section{Modelling and analysis of motion of a laboratory test rig}

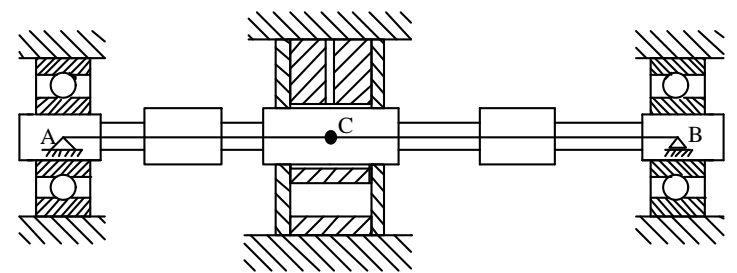

Fig. 6 A three-bearing rotor test rig

The techniques developed above have been employed to a laboratory test rig as shown in Fig. 6. The rig is a three-bearing rotor system. The rotor is two meters long and weighs $11.24 \mathrm{~kg}$. The active bearing is located $663 \mathrm{~mm}$ from the left ball bearing and 
$1037 \mathrm{~mm}$ from the right one. Some other parameters of the test rig are: bearing diameter $D=50 \mathrm{~mm}$; bearing length to diameter ratio $L / D=0.8$; bearing nominal radial clearance $c=0.3 \mathrm{~mm} ; \beta=105^{\circ}$ and $\Psi=160^{\circ}$ (see Fig. 1 and Fig. 2); thickness of the flexible sleeve $d=5 \mathrm{~mm}$; lubricant viscosity $\eta=0.04$ Pascal $\cdot$ Sec.

The rotor was modelled by the FEM with 26 elements and 104 DOF. The final condensed model contains only three rotor stations with only 6 DOF. The flexible sleeve was divided evenly into 20 elements with 60 DOF. The final condensed model contains only three sleeve stations with 3 DOF. It has been found that the final condensed models have almost identical eigenvalues and eigenvectors to the original FEM models up to the second mode for both the rotor and the flexible sleeve. The accuracy of the third eigenvalue is about $5 \%$ for the rotor and $6 \%$ for the flexible sleeve. The three eigenvalues of the condensed models are $10.43 \mathrm{~Hz}, 43.04 \mathrm{~Hz}$ and $106.38 \mathrm{~Hz}$ respectively for the rotor, and $490.2 \mathrm{~Hz}, 1754.2 \mathrm{~Hz}$ and $6175.8 \mathrm{~Hz}$ respectively for the flexible sleeve.

Both the non-linear model, Eq. 12, and the linearized model, Eq. 19, have been used to predict the thresholds of instability, critical speeds and unbalance response of the test rig in both the system configuration domain and the rotating speed domain. This paper only presents some simulation results when the chamber pressure in the active bearing was constant. In the system configuration domain analysis, the rotating speed was fixed to $50 \mathrm{~Hz}$. The active bearing position, relative to the reference line connecting the centres of two ball bearing, changes vertically only. In other words, the component of the configuration parameter $a_{x}$ is fixed to zero. It was defined that $a_{y}>0$, when the active bearing is above the line and $a_{y}<0$ if it is below the line. In the rotating speed domain analysis, the system configuration parameter was assumed to be zero, i.e. $a_{x}=a_{y}=0$.

\subsection{Equilibrium position calculations}

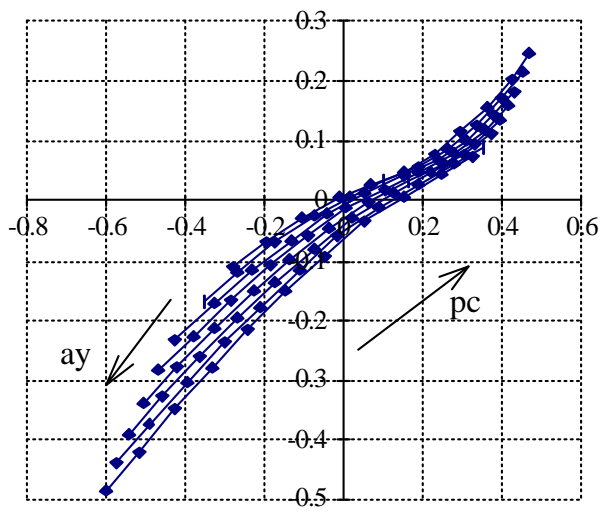

Fig. 7 Journal equilibrium position as a function of $a_{y}$ and $p_{c} ;\left(a_{y}=-1.0\right.$, $-0.5,0.0,0.5,1.0,1.5,2.0,2.5 \mathrm{~mm}$; $\left.p_{c}=0-0.25 \mathrm{MPa}\right)$

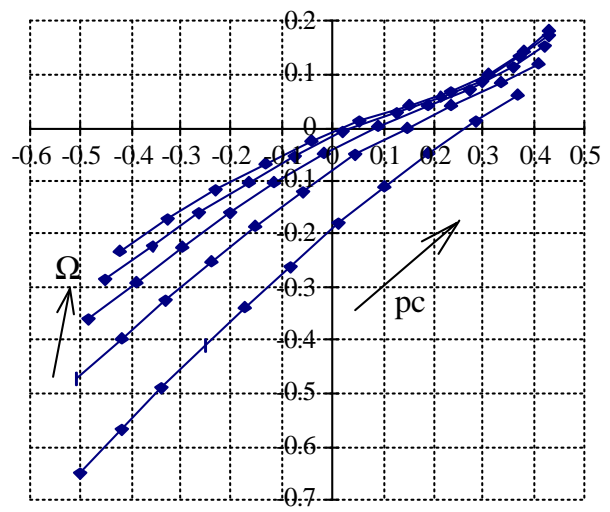

Fig. 8 Journal equilibrium position as a function of $\Omega$ and $p_{c},(\Omega=10,20,30$, 40 and $50 \mathrm{~Hz} ; p_{c}=0-0.25 \mathrm{MPa}$ )

Fig. 7 and Fig. 8 show the equilibrium position of the journal as functions of the chamber pressure in the configuration domain and the rotating speed domain respectively. (It is displayed in a non-dimensional form by dividing the actual values by the bearing nominal clearance). The equilibrium position was obtained by solving 
the static equation, Eq. 14, using the successive iteration method. By integrating nonlinear dynamic equation Eq. 12 in free vibration condition, the journal trajectory and hence the equilibrium position can also be obtained provided that the equilibrium position is stable. It has been found that the results, obtained from these two set of equations by the two different approaches, always agreed very well. Table 1 lists some examples of comparison between the results from the two methods when $a_{y}=-1.85 \mathrm{~mm}$ and $\Omega=50 \mathrm{~Hz}$. Solving Eq. 12 was very time consuming, whereas by using the successive iteration method, the iteration normally converged to an equilibrium very quickly.

\begin{tabular}{|l|l|l|l|l|}
\hline & \multicolumn{2}{|l|}{ from static model } & \multicolumn{2}{l|}{ from dynamic model } \\
\hline$p_{c}(\mathrm{MPa})$ & $\mathrm{x}$ & $\mathrm{y}$ & $\mathrm{x}$ & $\mathrm{y}$ \\
\hline 0.10 & 0.1583 & 0.0554 & 0.154 & 0.054 \\
\hline 0.15 & 0.2915 & 0.1234 & 0.286 & 0.120 \\
\hline 0.20 & 0.4096 & 0.2122 & 0.4060 & 0.2110 \\
\hline 0.25 & 0.4988 & 0.3023 & 0.4987 & 0.3023 \\
\hline
\end{tabular}

Table 4-1 Comparison of equilibrium position

\subsection{Prediction of thresholds of instability}

The stability of an equilibrium position can be assessed by either using the linearized model or the non-linear model. Eigenvalue analysis based on a linearized model is commonly used to study stability problems as well as to predict critical speeds. If the real part of one or more eigenvalues has positive value, the equilibrium position is unstable. In a non-linear model approach, the integration of the non-linear equations of motion for free vibration can produce trajectories of the rotor. If a trajectory rests on one point (an equilibrium position), the equilibrium position is stable. If a trajectory is in a limit cycle or in a chaos pattern, the equilibrium position is then unstable.

By solving the eigenvalue problems, the stability boundaries of the equilibrium position of the test rig were produced both in the system configuration domain and the rotating speed domain as shown in Fig. 9 and Fig. 10 respectively. If a combination of the configuration parameter and the chamber pressure, or the rotating speed and the chamber pressure, is located in the stable area, the corresponding equilibrium position is stable. Otherwise, it is unstable. The charts of stability boundaries provide such important information about the system, that one can select a value of chamber pressure to be above the pressure threshold of instability to ensure a stable equilibrium position.

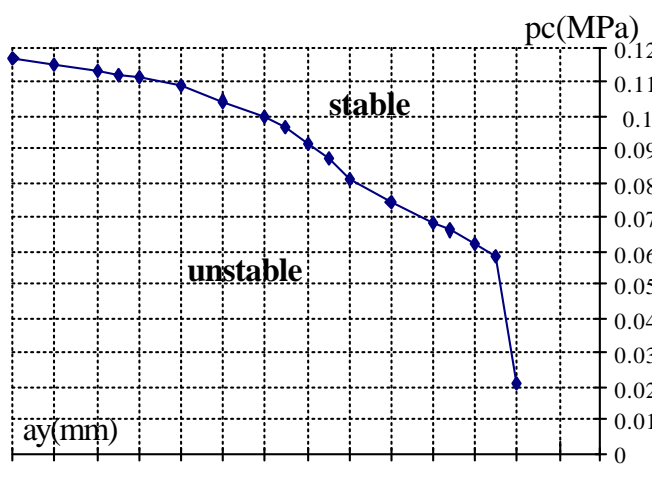

$-2.5-2.4-2.3-2.2-2.1-2-1.9-1.8-1.7-1.6-1.5-1.4-1.3-1.2-1.1$

Fig.9. Stability boundary in the configuration parameter domain

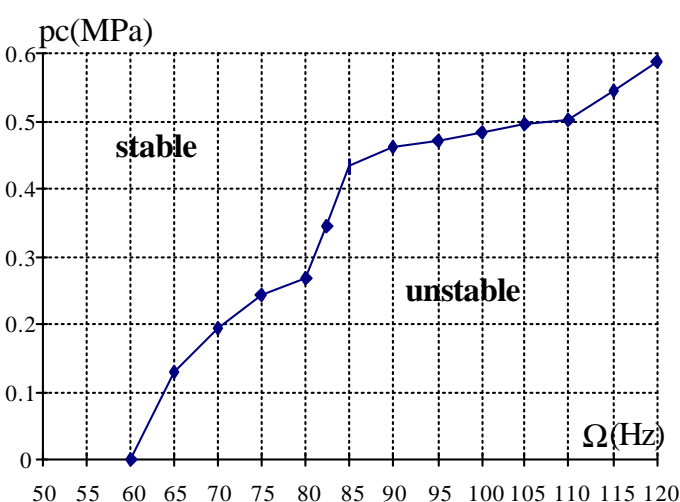

Fig.10. Stability boundary in the rotating speed domain 
To check the validity of the results obtained from the linearized model, numerical integration has been used to solve the free response of the system based on the nonlinear equations of motion. An example of such a numerical simulation is shown in Fig. 11, when $a_{y}=-1.85 \mathrm{~mm}, \Omega=50 \mathrm{~Hz}$, and $p_{c}=0.05 \mathrm{MPa}, 0.1 \mathrm{MPa}$ and $0.15 \mathrm{MPa}$ respectively. It is obvious that the journal performs a forward precession in a large limit cycle when $p_{c}=0.05 \mathrm{MPa}$, and its trajectory converges to a stable equilibrium quickly when $p_{c}=0.15 \mathrm{MPa}$. With $p_{c}=0.1 \mathrm{MPa}$, the trajectory approaches an equilibrium position very slowly. Hence, $0.1 \mathrm{MPa}$ could be considered as the threshold pressure of instability under this operation configuration. It is very close to the stability boundary obtained from the linearized model, which is $0.098 \mathrm{MPa}$ as shown in Fig. 9.

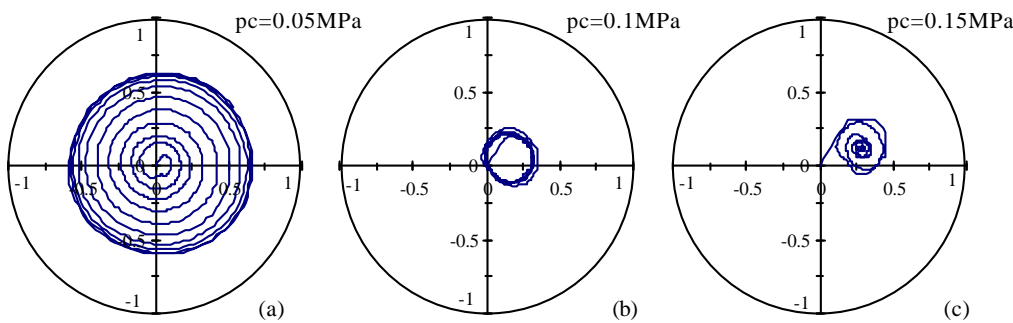

Fig. 11 Journal trajectories when $a_{y}=-1.85 \mathrm{~mm}, \Omega=50 \mathrm{~Hz}$

\subsection{Critical speed calculations}

Natural frequencies and critical speeds can be obtained from the imaginary parts of eigenvalues by eigenvalue analysis based on the linearized model. Fig. 12 shows the imaginary parts of eigenvalues (natural frequencies) as a function of rotating speed when the chamber pressure was fixed to $0.4 M P a$. (Two curves of natural frequencies corresponding to the flexible sleeve are not plotted in the chart because their values are too high to be fitted in the scale). The critical speeds of the rotor, read from the intersections of the dotted line $(\operatorname{Im}=\Omega)$ with the curves of the natural frequencies, are $39 \mathrm{~Hz}$ and $90 \mathrm{~Hz}$ respectively.

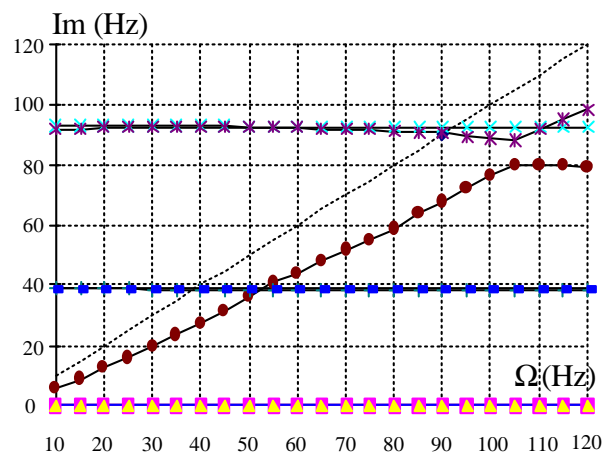

Fig. 12 Natural frequencies as a function of rotating speed

The critical speed can also be predicted by numerical simulations based on the nonlinear model. Fig. 13 displays a waterfall diagram of the unbalance response of the journal when $p_{c}=0.4 \mathrm{MPa}$. The diagram was obtained by numerical integration of the non-linear equation, Eq. 12. The synchronous harmonic of the unbalance response is shown in Fig. 14. The first and second critical speed, read from the graph, are $39 \mathrm{~Hz}$ and $91 \mathrm{~Hz}$ respectively which are very close to those from the eigenvalue analysis. It can be noticed that except the synchronous vibration, sub-harmonic and harmonic 
vibrations can also be observed from the non-linear simulation which cannot be obtained from the linearized model. It should be indicated that such a simulation of the waterfall diagram based on the non-linear model is very time consuming.

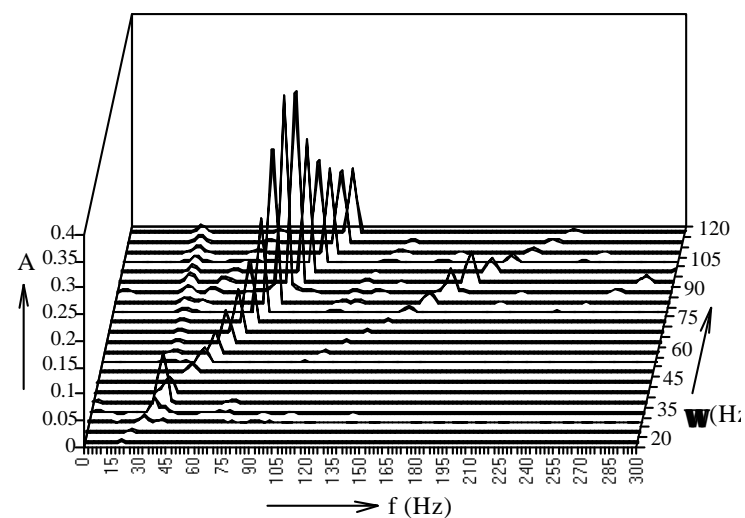

Fig. 13 Waterfall diagram of journal response

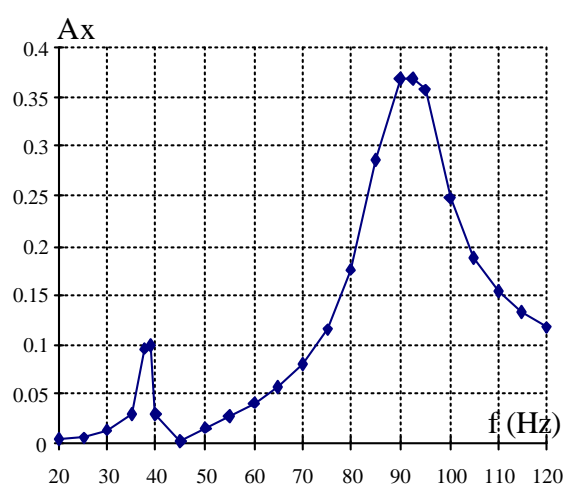

Fig. 14 Synchronous harmonic of journal response

\section{Conclusions}

The presented mathematical model can be used to predict dynamic behaviours of multi-bearing rotor systems incorporating the presented active journal bearing. Solutions obtained from the non-linear and linearized models are consistent. The linearized model is specially suitable for a overall investigation of the system characteristics over a wide range of system configuration parameters and the rotating speed in which numerous combinations of configuration parameters need to be analysed. Eigenvalue analysis based on the linearized model provides information of critical speeds and instability thresholds at the same time, while one sample solution of the non-linear equations of motion by numerical integration requires a considerable computing time. Numerical simulations based on the non-linear model provide not only a measure to verify the validity of results from the linearized model, it also provides some important information about the system, e.g. the details of the subharmonic whirling motion of the journal caused by the bearing oil film and limit cycles which cannot be obtained from the linearized model.

Computation time can be greatly reduced by employing the Guyan condensation technique. It actually made the solution by numerical integration of the non-linear equations of motion feasible in the calculations presented in this paper.

The developed non-linear and linearized equations of motion were also used in control law synthesis in forced vibration control by the proposed active journal bearng using both an open-loop approach and a feedback system. The results will be presented in other papers. 


\section{References}

1 - Z. A. PARSZEWSKI \& J. M. KRODKIEWSKI, Machine dynamics in term of the system configuration parameters, Proceedings of the International Conference on Rotor Dynamics, 1986, Tokyo, Japan, pp. 239-244.

2 - J. W. LUND, Stability and damped critical speeds of a flexible rotor in fluid film bearings, Trans. ASME, Journal of Engineering for Industry, 1974, Vol. 96, pp. 509517.

3 - M. L. ADAMS, Non-Linear Dynamics of Flexible Multi-Bearing Rotors, Journal of Sound and Vibration, 1980, Vol. 71, No. 1, pp. 129-144.

4 - G. SCHWEITZER, Magnetic bearings, Rotordynamics 2: Problems in turbomachinery, 1988, Springer Verlag Wien-New York, pp. 543-570.

5 - A. B. PALAZZOLO, R. R. LIN, R. M. ALEXANDER, A. F. KASCAK, \& J. MONTAGUE, Test and theory for piezoelectric actuator-active vibration control of rotating machinery, Trans. ASME, Journal of Vibration and Acoustics, 1991, Vol. 113, pp. 167-175.

6 - H. ULBRICH \& J. ALTHAUS, Actuator design for rotor control, The 1989 ASME Design Technical Conference, 12th Biennial Conference on Mechanical Vibration and Noise, 1989, Montreal, Quebec, Canada, pp. 17-22.

7 - J. ALTHAUS \& H. ULBRICH, A fast hydraulic actuator for active vibration control, IMechE 1992, C432/045, pp. 141-148.

8 - G. P. FENG, \& N. XIN, Automatic control of the vibration of the flexible rotor with microcomputer, Proceedings of International Conference on Rotordynamics, Tokyo, 1986, pp. 443-448.

9 - M. J. GOODWIN, J. E. T. PENNY \& C. J., HOOKE, Variable impedance bearings for turbogenerator rotors, IMechE, 1984, C288/84, pp. 535-541.

10 - M. J. GOODWIN, M. P. ROACH \& J. E. T. PENNY, An analysis of combined squeeze-film and variable stiffness hydrostatic bearings, and their use in aircraft engine vibration control, Vibrations in Rotating Machinery, IMechE, Heriot-Watt University, 1988, Edinburgh, C282/88, pp. 85-91.

11 - J. L. NIKOLAJSEN, \& M. S. HOQUE, An electroviscous damper, proceedings of nato/asi conference, Vibration Wear Damage High Speed Rotating Machinery, 1989, Troia Beach, Lisbon.

12 - J. KICINSKI \& P.MATERNY, Non-linear vibrations in multi degree freedom system on the example of turbine $13 \mathrm{k} 215$, Proceedings of the International Conference on Vibration and Noise, 1995, Venice, pp. 120-127.

13 - J. M. KRODKIEWSKI \& L. SUN, Stability control of rotor-bearing system by an active journal bearing, Proceedings of the International Conference on Vibration and Noise, 1995, Venice, pp. 217-225.

14 - R. J. GUYAN, Reduction of stiffness and mass matrices, American Institute of Aeronautics and Astronautics Journal, 1965, Vol. 3, No. 2, pp. 380.

15 - B. CARNAHAN, H. A. LUTHER, \& H. O. WILKES, Applied numerical methods, 1969, John Wiley \& Sons, New York.

16 - J. F. TRAUB, Iterative methods for the solution of equations, Prentice-Hall, Englewood Cliffs, 1964, New Jersey. 